\title{
Developing a Telemedicine Curriculum for a Family Medicine Residency
}

Emmeline Ha, MD | Kristen Zwicky, MD | Grace Yu, MD | Andrew Schechtman, MD

PRiMER. 2020;4:21.

Published: 9/21/2020 | DOI: 10.22454/PRiMER.2020.126466

\section{Abstract}

Introduction: Telemedicine has rapidly become an essential part of primary care due to the COVID-19 pandemic. However, formal training in telemedicine during residency is lacking. We developed and implemented a telemedicine curriculum for a family medicine residency program and investigated its effect on resident confidence levels in practicing telemedicine.

Methods: We designed a process map of the telemedicine visit workflow at the residency clinic to identify key topics to cover in the curriculum. We created a live 50-minute didactic lecture on best practices in telemedicine, along with a quick-reference handout. We distributed pre- and postintervention surveys to current residents $(\mathrm{N}=24)$ to assess the effect of the educational intervention on their confidence in practicing telemedicine.

Results: Fourteen residents ( $58 \%$ response rate) completed all aspects of the study including both surveys and participation in the educational intervention. Confidence levels in conducting telemedicine visits increased in three of five domains: (1) virtual physical exam $(P=.04)$, (2) visit documentation ( $P=.03)$, and (3) virtually staffing with an attending $(P=.04)$. Resident interest in using telemedicine after residency also increased following the educational intervention.

Conclusion: Telemedicine requires a unique skill set. Formal education on best practices improves resident confidence levels and interest in practicing telemedicine. Primary care residency programs should incorporate telemedicine training to adequately prepare their graduates for clinical practice.

\section{Introduction}

Telemedicine is the practice of using technology to deliver health care remotely. ${ }^{1}$ While it has been a growing industry for the last 2 decades, widespread adoption was limited by reimbursement restrictions and technology barriers. The need for telemedicine abruptly increased during the 2020 COVID-19 pandemic because it served as a method for continuing health care while limiting in-person exposures. In graduate medical education, telemedicine was rapidly adopted once the Centers for Medicare and Medicaid Services expanded reimbursement for telemedicine outside of rural areas and allowed virtual precepting of resident visits via video/phone. ${ }^{2}$

There are several nuances to practicing telemedicine that require unique skills compared to in-person care. ${ }^{3-5}$ For example, visit logistics change when incorporating technology. Maintaining professionalism requires greater consideration of the surrounding environment and body language. The physical exam involves coaching patients to self-perform certain maneuvers. These aspects are different enough that formal training is essential to providing high-quality care through telemedicine. Despite this, there is a lack of training in telemedicine in primary care 
residency programs, likely due to low utilization prior to the COVID-19 pandemic. In a 2015 survey of 207 family medicine residencies nationwide, the majority of program directors reported that they had telehealth services in some form, but actual use was limited and infrequent. ${ }^{6}$

While the American Academy of Family Physicians endorses telemedicine exposure during residency, there is no recognized gold standard for training in graduate medical education. ${ }^{1,7}$ There are several studies on telemedicine education in other specialties; however, to our knowledge there are no published studies in family medicine ${ }^{8-14}$ The Society of Teachers of Family Medicine (STFM) perceives a need for standardized training and recently created a task force to develop a national telemedicine curriculum. ${ }^{1}$ In this study, we developed a telemedicine curriculum for the Stanford-O'Connor Family Medicine Residency Program and investigated its impact on resident confidence and attitudes toward telemedicine.

\section{Methods}

The study population included current Stanford-O'Connor family medicine residents $(\mathrm{N}=24)$, whose clinic is an urban federally qualified health center. To develop the educational intervention, we designed a process map of our telemedicine visit workflow to identify key topics to include (Figure 1). We then developed a 50-minute lecture that was conducted live during regularly-scheduled didactic time. The lecture was recorded and made available to all residents, along with a quick-reference handout. Topics included appropriate visit types, logistics, technology set-up, etiquette, effective communication, virtual physical exam, documentation, and staffing with an attending. These resources are available for public access on the STFM Resource Library. ${ }^{15}$

Residents completed a pre- and postintervention survey on their confidence in conducting various aspects of a telemedicine visit using a 4 -point Likert scale ( $0=$ not at all confident, $4=$ very confident). They were also assessed on their attitude toward telemedicine by indicating their level of agreement with different statements. To be included in the study, residents were required to complete both pre- and postsurveys and the educational intervention. Survey data were linked by deidentified participant ID numbers, and statistical significance was determined by the pairedsample Wilcoxon signed rank test. The Stanford University School of Medicine Institutional Review Board granted this project exemption.

\section{Results}

Of the 24 current residents, 14 (58\%) met inclusion criteria. Resident mean confidence levels in conducting telemedicine visits increased in all domains (Table 1). Three domains were statistically significant $(P<.05)$ : virtual physical exam (median 1 vs 2), documenting the visit (median 2.5 vs 3), and virtually staffing with an attending (median 3 vs. 4). Confidence levels for the logistics of a telemedicine visit (median 2 vs 2 ) and taking a history through telemedicine (median 3 vs 3 ) did not significantly increase. Following the intervention, all residents expressed interest in using telemedicine after residency (Figure 2).

\section{Conclusions}

We developed and implemented a telemedicine curriculum for family medicine residents that included a didactic lecture and quick-reference handout. Prior to our educational intervention, most residents felt comfortable obtaining a history through telemedicine, but confidence was lower for conducting a virtual physical exam, documenting, and virtually staffing with an attending. The results of our study show significant increases in confidence levels in these domains after formal telemedicine education. Confidence in logistics for a telemedicine visit did not change, likely because residents were already familiar with the workflow standardized by clinic administration before our curriculum was implemented. Our findings are consistent with prior studies on resident telemedicine training in a variety of specialties. ${ }^{8-13}$ We are the first, to our knowledge, to report such findings for family medicine residents.

Limitations of our study include self-reported data, selection bias from voluntary survey participation, and small 
sample size due to inclusion of a single site. Additionally, the time between educational intervention and postsurvey was only 1 week. This short interval was chosen to prevent residents from having significant time to complete more telemedicine visits, which could have confounded our data. Assessing the durability of these confidence increases over time was not within the scope of our study. Finally, it is unclear what is the best method of telemedicine instruction in graduate medical education, given the lack of a current gold standard for training.

Our educational resources are publicly available to assist other residency programs in developing their own telemedicine curriculum. Future directions include expanding our study to multiple sites to determine if findings are generalizable to a larger population. Additionally, longitudinal outcomes could be assessed to determine if a onetime educational intervention creates lasting effects on resident skills and attitudes toward telemedicine.

While the future of telemedicine post-COVID-19 remains unclear, current widespread use and advocacy for permanent expansion of reimbursement indicates that it will continue to have an important role in health care. Given this, primary care residency programs should incorporate telemedicine training to adequately prepare their graduates for clinical practice. Our developed curriculum contributes to the growing body of telemedicine education in residency.

\section{Tables and Figures}

Figure 1: Process Map of Telemedicine Visit Workflow at StanfordO’Connor Family Medicine Residency Clinic

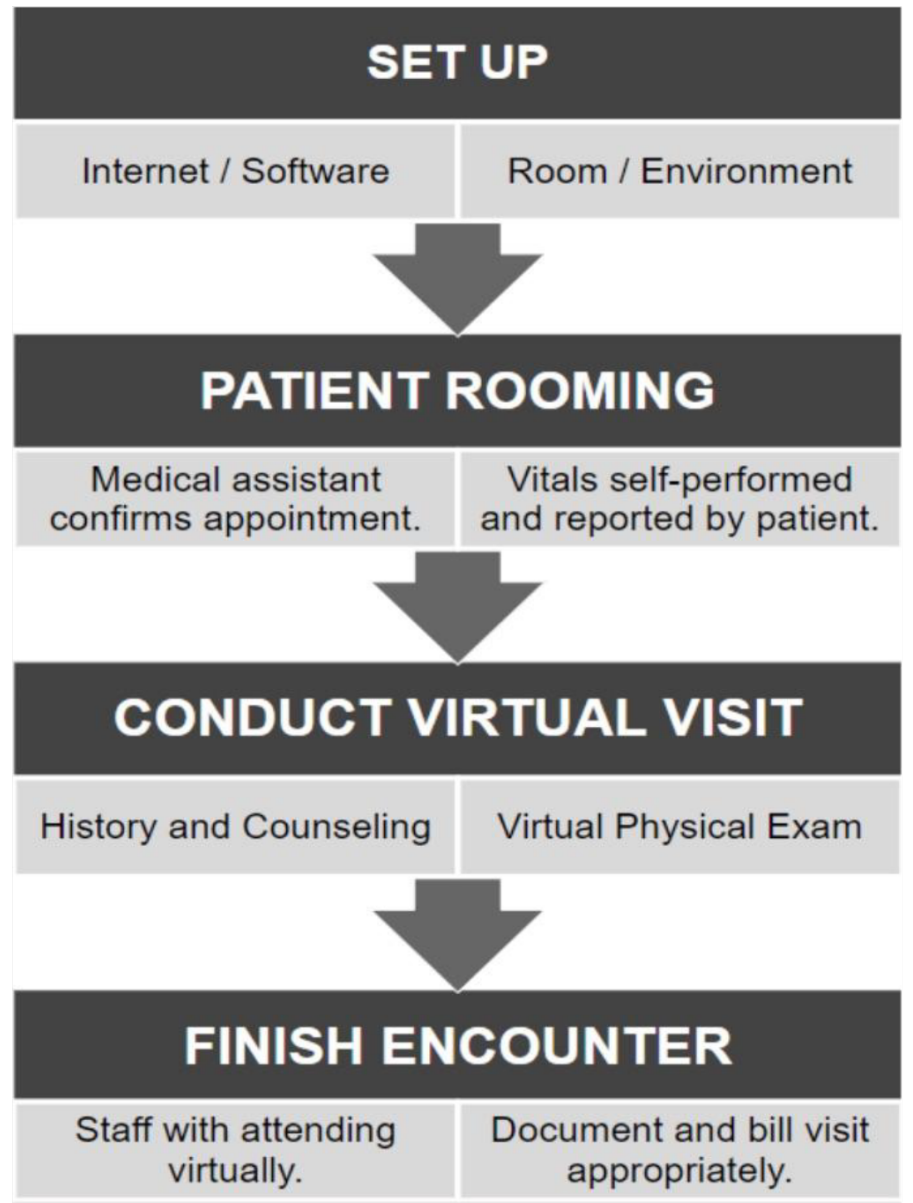


Figure 2: Pre- and Posteducational Intervention Resident Responses to "After

Residency, I Am Interested in Using Telemedicine in My Clinical Practice."

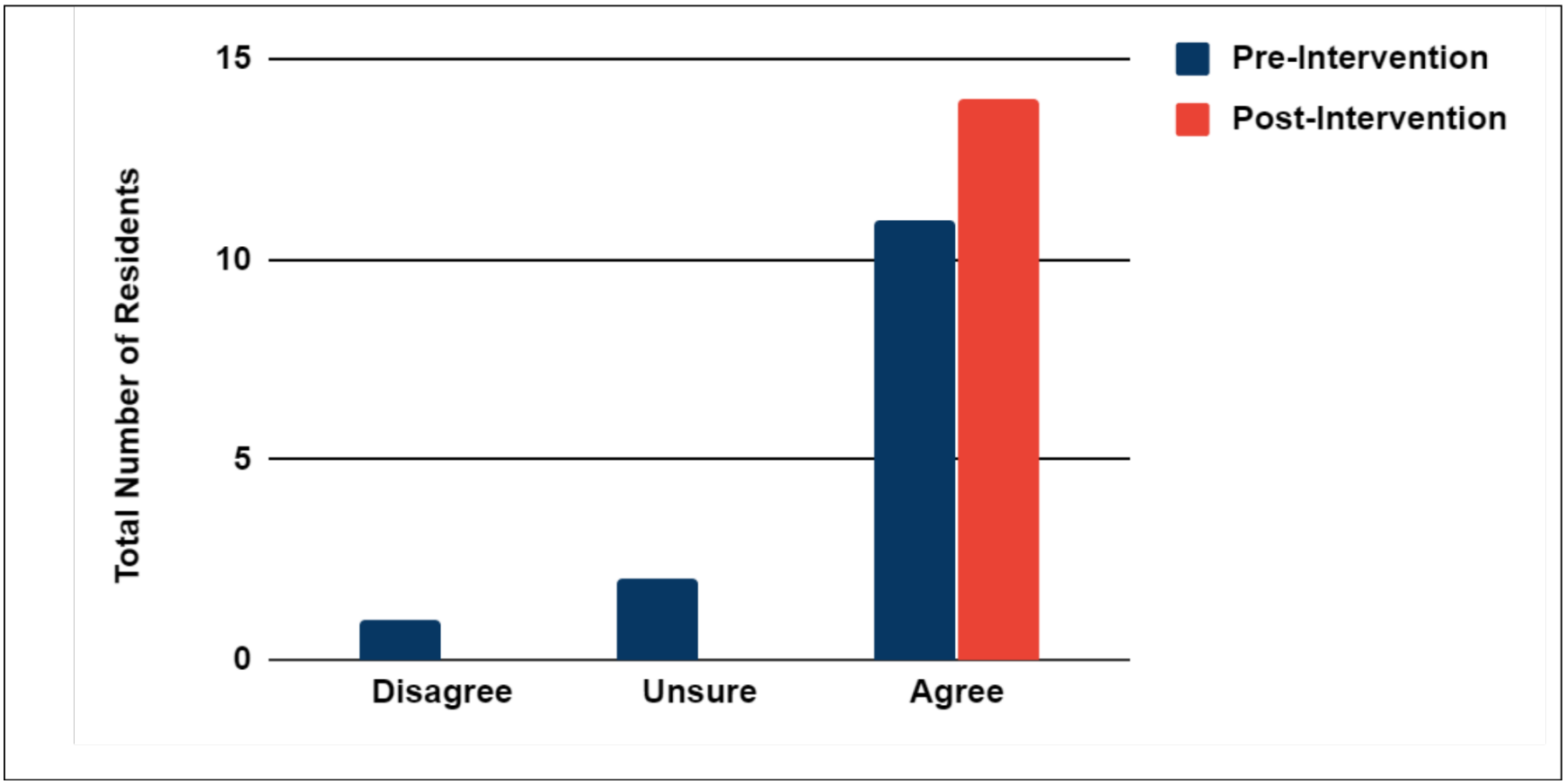

Table 1: Reported Resident Confidence Levels in Telemedicine Visit Domains, Pre- and Posteducational Intervention ( $0=$ Not at All Confident, $1=$ Less Confident, 2=Somewhat Confident, 3=Very Confident)

\begin{tabular}{|l|l|l|l|l|l|}
\hline \multirow{2}{*}{ Domain } & \multicolumn{2}{|c|}{ Preintervention Confidence } & \multicolumn{2}{c|}{ Postintervention Confidence } & \multicolumn{1}{c|}{ M Value $^{\text {a }}$} \\
\cline { 2 - 5 } & \multicolumn{1}{|c|}{ Mean (SD) } & Median & $2.43(0.51)$ & 2 & .2 \\
\hline $\begin{array}{l}\text { Logistics of a telemedicine } \\
\text { visit }\end{array}$ & $2.14(0.36)$ & 2 & $2.93(0.27)$ & 3 & .5 \\
\hline $\begin{array}{l}\text { Taking a history through } \\
\text { telemedicine }\end{array}$ & $2.79(0.43)$ & 3 & $2(0.68)$ & 2 & .04 \\
\hline $\begin{array}{l}\text { Conducting a virtual physical } \\
\text { exam }\end{array}$ & $1.21(0.97)$ & 1 & $2.5(0.65)$ & 3 & .03 \\
\hline $\begin{array}{l}\text { Documenting a telemedicine } \\
\text { visit }\end{array}$ & $1.79(0.89)$ & 2.5 & $2.21(0.7)$ & 4 & .04 \\
\hline $\begin{array}{l}\text { Staffing with an attending } \\
\text { virtually }\end{array}$ & $1.57(0.65)$ & 3 & & \\
\hline
\end{tabular}

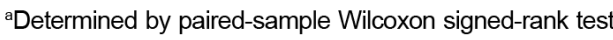

\section{Acknowledgments}

Drs Ha and Zwicky served as co-first authors and contributed equally to the work.

\section{Corresponding Author}

Emmeline $\mathrm{Ha}, \mathrm{MD}$

455 O'Connor Drive, Suite 250, San Jose, CA 95128. 408-283-7767. Fax: 408-283-7608

\section{Author Affiliations}

Emmeline Ha, MD - Stanford-O'Connor Family Medicine Residency, San Jose, CA

Kristen Zwicky, MD - Stanford-O'Connor Family Medicine Residency, San Jose, CA 
Grace Yu, MD - Stanford-O'Connor Family Medicine Residency, San Jose, CA

Andrew Schechtman, MD - Stanford-O'Connor Family Medicine Residency, San Jose, CA

\section{References}

1. Theobald M, Brazelton T. STFM forms task force to develop a national telemedicine curriculum. Ann Fam Med. 2020;18(3):285-286. doi:10.1370/afm.2549

2. Centers for Medicare and Medicaid Services. Teaching Hospitals, Teaching Physicians and Medical Residents: CMS Flexibilities to Fight COVID-19. July 2020. Baltimore, MD: Centers for Medicare \& Medicaid Services. https://www.cms.gov/files/document/covid-teaching-hospitals.pdf. Accessed September 11, 2020.

3. Onor ML, Misan S. The clinical interview and the doctor-patient relationship in telemedicine. Telemed J E Health. 2005;11(1):102-105. doi:10.1089/tmj.2005.11.102

4. Allen DT, Caldwell P, Komesaroff PA, et al; Royal Australasian College of Physicians Telehealth Working Group. Practical aspects of telehealth: set-up and preparation for video consultations. Intern Med J. 2013;43(10):1133-1136. doi:10.1111/imj.12264

5. Bulik RJ. Human factors in primary care telemedicine encounters. J Telemed Telecare. 2008;14(4):169-172. doi:10.1258/jtt.2007.007041

6. Moore MA, Jetty A, Coffman M. Over half of family medicine residency program directors report use of telehealth services. Telemed J E Health. 2019;25(10):933-939. doi:10.1089/tmj.2018.0134

7. American Academy of Family Physicians. Recommended Curriculum Guidelines for Family Medicine Residents Medical Informatics. August 2018. Leawood, KS: American Academy of Family Physicians; August 2018. https://www.aafp.org/dam/AAFP/documents/medical_education_residency/program_directors /Reprint288_Informatics.pdf. Accessed September 11, 2020.

8. Fleming DA, Riley SL, Boren S, Hoffman KG, Edison KE, Brooks CS. Incorporating telehealth into primary care resident outpatient training. Telemed J E Health. 2009;15(3):277-282. doi:10.1089/tmj.2008.0113

9. Kirkland EB, DuBose-Morris R, Duckett A. Telehealth for the internal medicine resident: A 3-year longitudinal curriculum [published online ahead of print, 2019 Dec 31]. J Telemed Telecare. 2019;1357633X19896683. doi:10.1177/1357633X19896683

10. Sunderji N, Crawford A, Jovanovic M. Telepsychiatry in graduate medical education: a narrative review. Acad Psychiatry. 2015;39(1):55-62. doi:10.1007/s40596-014-0176-x

11. Marttos AC Jr, Fernandes Juca Moscardi M, Fiorelli RKA, et al. Use of telemedicine in surgical education: a seven-year experience. Am Surg. 2018;84(8):1252-1260. doi:10.1177/000313481808400831

12. Afshari M, Witek NP, Galifianakis NB. Education research: an experiential outpatient teleneurology curriculum for residents. Neurology. 2019;93(4):170-175. doi:10.1212/WNL.0000000000007848

13. Summe A, Foor L, Hoeck L, Campbell K, Boyd M, Barnes MA. Resident perceptions of competency and comfort before and after telemedicine-ICU implementation. South Med J. 2018;111(6):344-347. doi:10.14423/SMJ.0000000000000813

14. Wanat KA, Newman S, Finney KM, Kovarik CL, Lee I. Teledermatology education: current use of teledermatology in US residency programs. J Grad Med Educ. 2016;8(2):286-287. doi:10.4300/JGMED-16-00041.1

15. Zwicky K, Ha E. Telemedicine 101: Best Practices for Residents. June 3, 2020. STFM Resource Library. https://resourcelibrary.stfm.org/viewdocument/telemedicine-101-best-practicesfo?CommunityKey=2751b51d-483f-45e2-81de-4faced0a290a. Accessed September 11, 2020.

Copyright $\odot 2020$ by the Society of Teachers of Family Medicine 